\title{
A retrospective study on analgesic requirements for thoracoscopic surgery postoperative pain
}

\section{Hiroaki Kuroda' \\ Hitomi Mizuno \\ Hitoshi Dejima' \\ Kiyoe Watanabe ${ }^{2}$ \\ Tatsuya Yoshida ${ }^{3}$ \\ Yumiko Naito \\ Yukinori Sakao'}

'Department of Thoracic Surgery, ${ }^{2}$ Department of Nursing, ${ }^{3}$ Department of Thoracic Oncology, Aichi Cancer Center Hospital, Nagoya, Japan
Correspondence: Hiroaki Kuroda Department of Thoracic Surgery, Aichi Cancer Center Hospital, I-I, Kanokoden, Chikusa-ku, Nagoya 464-868I, Japan

Tel +8I 527626 III

Fax +8I 527635233

Email h-kuroda@aichi-cc.jp
This article was published in the following Dove Press journal: Journal of Pain Research

15 November 2017

Number of times this article has been viewed

Background: Thoracoscopic surgery (TS) has been performed as a minimally invasive procedure since the beginning of the 1990s. This has led to a dramatic change in the postoperative condition of these patients, facilitating early ambulation and easier management of postoperative pain. However, empirical evidence on postoperative pain management after TS is limited. The aims of this study were to determine the efficacy and adequacy of postoperative analgesic medications and to simplify the choice of additional drugs based on a numerical rating scale (NRS). Materials and methods: A retrospective study of patients who underwent TS was performed to evaluate postoperative pain, analgesia requirements, and the number of drugs needed during the perioperative period based on the NRS score.

Results: Of the 524 patients, mild pain was noted in $87 \%$ patients on the day of the operation and in $75.6 \%$ patients on ambulation. The mean NRS score was $1.83 \pm 1.49$ on the day of the operation and $2.73 \pm 1.75$ on ambulation. An NRS score of 3 on both the day of operation and on ambulation was defined as the necessary condition for improved pain management. Multivariate analysis showed that high surgical stress significantly influenced pain scores. Reduction in pain with an NRS score of $\geq 1$ was significant with the addition of pentazocine hydrochloride $(p<0.01)$ and flurbiprofen $(p<0.01)$. Interestingly, the addition of tramadol was borderline efficacious $(p=0.05)$ in patients with an NRS score of $>3$ on ambulation.

Conclusion: A small number of patients have moderate-to-severe pain after TS. Tramadol demonstrated borderline efficacy in controlling postoperative intense pain with an NRS score of $\geq 3$.

Keywords: tramadol, thoracoscopic surgery, ambulation, NRS, postoperative pain

\section{Introduction}

Early ambulation after thoracoscopic surgery (EATS) based on the concepts of enhanced recovery after surgery (ERAS) was introduced to our institution from June 2013 with the dual purpose of improving patient confidence in perioperative restoration and preventing postoperative complications. ${ }^{1}$ Although the clinical outcomes of pain management after surgery were technically influenced by EATS, which has been widely studied, there is a paucity of relevant information on early and definite pain management in the ward. This includes postoperative analgesic requirements, adequate number of analgesic medications, and the efficacy of each drug for further pain management. ${ }^{2,3}$

The current tendency to use non-opioid drugs for perioperative analgesia as a substitute for opioids has been emphasized. ${ }^{4-6}$ Opioid-related adverse events contribute to the failure of early ambulation and delayed discharge. ${ }^{4}$ Interestingly, some authors report that thoracoscopic surgery (TS) could be safely performed in lung cancer lobectomy 
patients and seemed to reduce postoperative complications and length of hospital stay. ${ }^{7}$ Das-Neves-Pereira et al $^{7}$ reported that their analgesia method for first-track rehabilitation was the oral administration of opioid-free analgesic drugs in combination with thoracic epidural patient-controlled analgesia. In our EATS program, we usually manage postoperative pain by intraoperative intercostal nerve block alone. Oral administration of opioid-free medications was minimized during the perioperative period.

A detailed analysis of analgesia drugs for patients who underwent TS has not been described. In this study, the primary aim was to investigate the most effective analgesia drugs in the clinical course of our population and to simplify the choice of alternative medications corresponding to a numerical rating scale (NRS) score.

\section{Materials and methods EATS}

In wards, EATS is worth the early physical exercise. Once the patient is fully awake, subsequently pain and vital signs are under control, EATS protocol is started 2-4 hours after operation. Then, patients are accommodated in seated position and immediately encouraged the physical exercises and deep breathing for 10 minutes. If patients had no severe symptoms, the patients were stimulated to walk around the thoracic surgical ward with the nursing staff and thoracic surgeons. The sufficient pain management could play a key role in EATS.

\section{Patients}

We performed a retrospective analysis on 524 patients who underwent TS at Aichi Cancer Center between June 2013 and March 2016. This series included consecutive patients who were preoperatively selected for inclusion to the EATS protocol. The selection criteria for EATS included TNM clinical stage IA lung cancers, pulmonary metastasis, any lung cancer stage in a patient $>75$ years old, can possibly tolerate general anesthesia, and provision of informed consent for EATS. ${ }^{8}$ Patients were followed-up for 4 days after the TS in order to evaluate the adequacy of postoperative pain management. Patient's pain was evaluated using NRS graded from 0 to 11 (the worst pain imaginable). Serial monitoring (eight to 12 per day) of NRS score was assessed through completion of the questionnaire by nursing staff in the thoracic specific ward. These data were especially drawn a parallel before and after the analgesics administered postoperatively, on ambulation in the wards, and until discharge.
Because individual patients were not identified, the institutional review board of Aichi Cancer Center approved this study without the requirement of obtaining informed consent (No. 2016-1-051). The patient records and information were anonymized prior to analysis.

\section{Analgesics protocols}

\section{Intraoperative course}

Intraoperative intercostal nerve block using ropivacaine hydrochloride hydrate $(0.2 \%$ at $20 \mathrm{mg} / \mathrm{kg}$ body weight $)$ was performed without epidural anesthesia or paravertebral block (PVB). ${ }^{9}$

\section{Postoperative course}

Following extubation and awakening from anesthesia, patients were transferred to the thoracic specific ward where, according to the defined protocol to use analgesics by the thoracic surgical team, the nursing staff administered oral loxoprofen $180 \mathrm{mg} /$ day after the confirmation of reliable oral intake immediately. Additional analgesics as following based on repeated assessment of NRS pain scores were provided by the judgments of nursing staff, if the patients wanted to reduce the postsurgical pain: intravenous drip (pentazocine [15-30 mg/day], flurbiprofen [50-150 mg/day]), oral administrations (tramadol [25-100 mg/day] and pregabalin [75-150 mg/day]), and suppository (diclofenac [50-200 mg/ day]). For the requirement of the further painkilling release, the nursing staff corresponded to the dose escalation of previous medication or an additional new analgesic for synergy effect (Figure 1).

This variety of management choices potentially detracts from the reliability of the results regarding pain scores and rate of usage of additional analgesics. Therefore, the aims of this retrospective study were to determine the efficacy and adequacy of postoperative analgesic medications and to clarify the most effective additional analgesics based on an NRS.

\section{Statistical analysis}

Data were analyzed using SPSS software (version 17.0; SPSS Inc., Chicago, IL, USA). We investigated the NRS score to identify the ideal necessary situation of more than two analgesic drugs using receiver operating characteristic (ROC) curves and measured areas under the receiver operating characteristic curves (AUROCs). Differences between two groups were assessed using the Mann-Whitney $U$-test. Multiple logistic regression analysis was performed to 


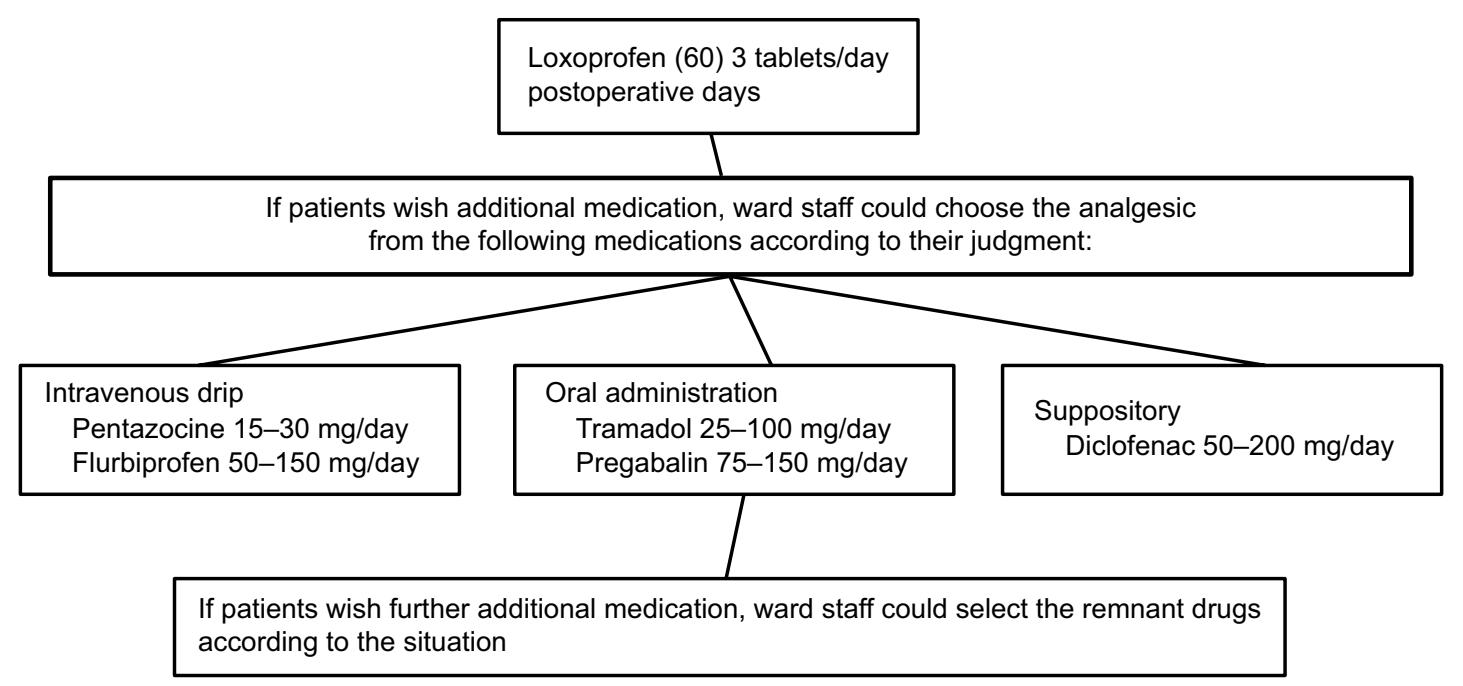

Figure I Postoperative course of postoperative pain after TS in our hospital. Abbreviation: TS, thoracoscopic surgery.

assess the effect of several factors on postoperative pain. Statistical significance was considered to be achieved at $p<0.05$.

\section{Results}

The clinical characteristics of the 524 study patients are summarized in Table 1. The median age was 66 years (range, 19-87 years), and the proportion of men was 52.1\% $(273 / 524)$. The surgical procedures performed were lobectomy and segmentectomy in $67.6 \%$ patients (four-port TS, maximum incision of $30 \mathrm{~mm}$ ) and wedge and mediastinal tumor resection in $32.4 \%$ patients (three-port TS, maximum incision of $20 \mathrm{~mm}$ ). Mean operative time was $183 \pm 81 \mathrm{~min}-$ utes; $11.6 \%$ of patients had $>1 \mathrm{~mL}$ of bleeding per kilogram body weight.

Individual pain scores were classified as no and mild $(0-3 / 10)$, moderate $(4-6 / 10)$, and severe (7-10/10). Incidence of mild pain was $87.0 \%(n=456)$ on the day of operation, $75.6 \%$ upon ambulation, and $89.9 \%$ on settling $(\mathrm{n}=471$; Figure 2A). Mean NRS score was $1.83 \pm 1.49$ on the day of operation, $2.73 \pm 1.75$ upon ambulation, and $1.87 \pm 1.33$ on settling, after several analgesic drugs. In our hospital, all patients who underwent open thoracotomy received PVB. However, the mean NRS score was $3.05 \pm 1.51$ upon ambulation in the patients with open thoracotomy, which was significantly higher than in those with TS $(p<0.01)$.

Further investigation of the optimal cutoff value of NRS score that necessitated administration of a third analgesic drug was performed. As shown in Figure 2B, the AUROCs was 0.69 (95\% CI: $0.64-0.75)$ on the day of operation, 0.74
Table I Patient characteristics

\begin{tabular}{|c|c|}
\hline Variables $(n=524)$ & Value \\
\hline Age (years) & $65.5 \pm$ II.3 (19-87) \\
\hline \multicolumn{2}{|l|}{ Mean $\pm S D$ (range) } \\
\hline \multicolumn{2}{|l|}{ Male/female } \\
\hline Ratio & $273 / 25 \mid(52.1 \%)$ \\
\hline \multicolumn{2}{|l|}{ Respiratory function } \\
\hline$\leq 70 />70$ (ratio) & $54 / 470(10.3 \%)$ \\
\hline \multicolumn{2}{|c|}{ Procedure/operative time } \\
\hline \multicolumn{2}{|c|}{ Lobectomy/segmentectomy, operative } \\
\hline \multicolumn{2}{|l|}{ time (minutes) } \\
\hline Ratio & $354(67.6 \%)$ \\
\hline Mean \pm SD (range) & $224.2 \pm 60.7(59-487)$ \\
\hline \multirow{2}{*}{\multicolumn{2}{|c|}{$\begin{array}{l}\text { Wedge/mediastinal resection, operative } \\
\text { time (minutes) }\end{array}$}} \\
\hline & \\
\hline Ratio & $170(32.4 \%)$ \\
\hline Mean \pm SD (range) & $99.9 \pm 46.2(34-323)$ \\
\hline \multicolumn{2}{|l|}{ Body mass index } \\
\hline Mean \pm SD (range) & $22.4 \pm 3.6(19.7-44.1)$ \\
\hline \multicolumn{2}{|l|}{ Bleeding/body weight } \\
\hline$>I / \leq I$ (ratio) & $6 \mathrm{I} / 463(1 \mathrm{I} .6 \%)$ \\
\hline \multicolumn{2}{|c|}{ Placement of drainage tube } \\
\hline Mean \pm SD (range) & $1.3 \pm 1.4(0-12)$ \\
\hline
\end{tabular}

(95\% CI: 0.69-0.78) upon ambulation, and 0.63 (95\% CI: $0.57-0.68$ ) on settling after medications.

There was a moderate correlation between number of necessary analgesic drugs and NRS on ambulation $(r=0.451$, $p<0.01$; Figure 3A). Reduction in pain with NRS score $\geq 1$ was significant with the addition of pentazocine hydrochloride $(p<0.01)$ and flurbiprofen $(p<0.01$; Figure 3B). In addition, we identified the most effective additional analgesic drug for the 253 patients (47.8\%) with NRS score of $\geq 3$ upon ambulation. Significant differences were observed in the efficacy of desirable pain control regarding dramatic NRS 
A

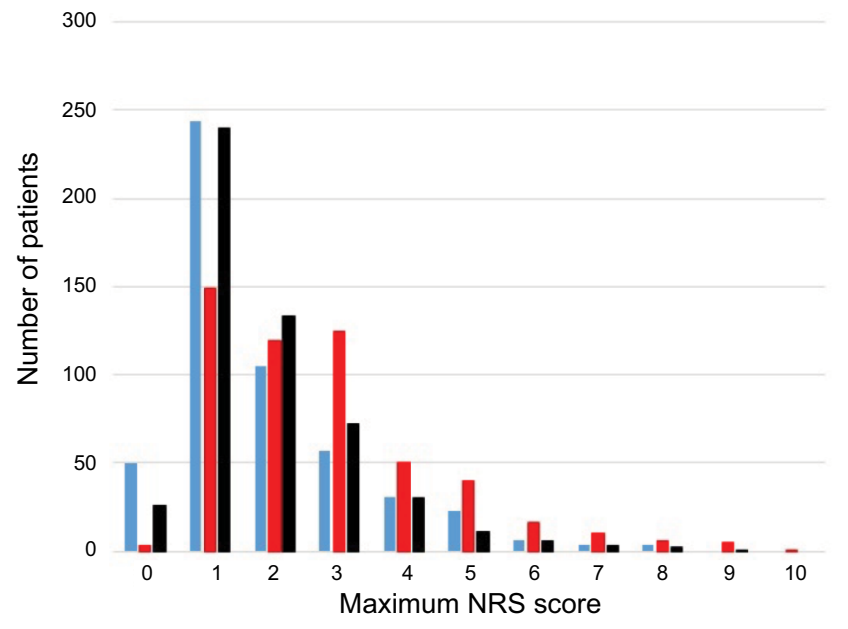

B

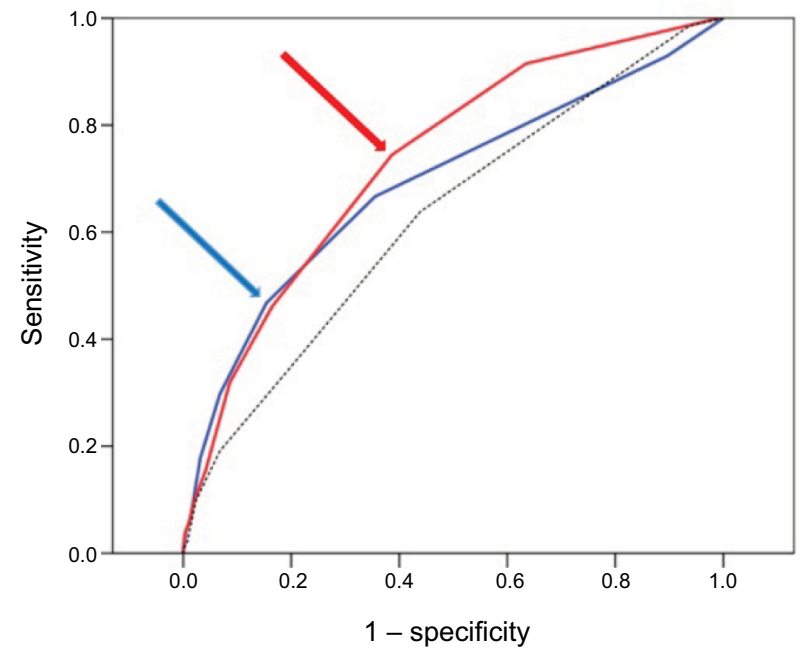

Figure 2 Postoperative pain after TS.

Notes: (A) Distribution of the maximum NRS score for pain on postoperative day (blue), upon ambulation (red), and on settling after medications (black). (B) ROC curve analyses for pain on postoperative day (blue), upon ambulation (red), and on settling after medications (black dotted). Arrows indicate the cutoff values.

Abbreviations: TS, thoracoscopic surgery; NRS, numerical rating scale; ROC, receiver operating characteristic.

A

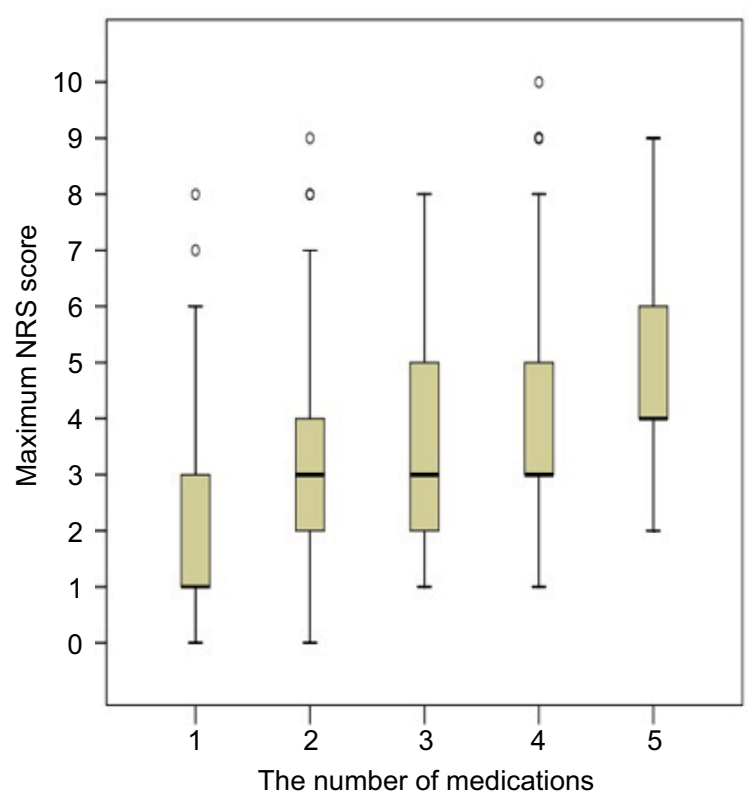

B

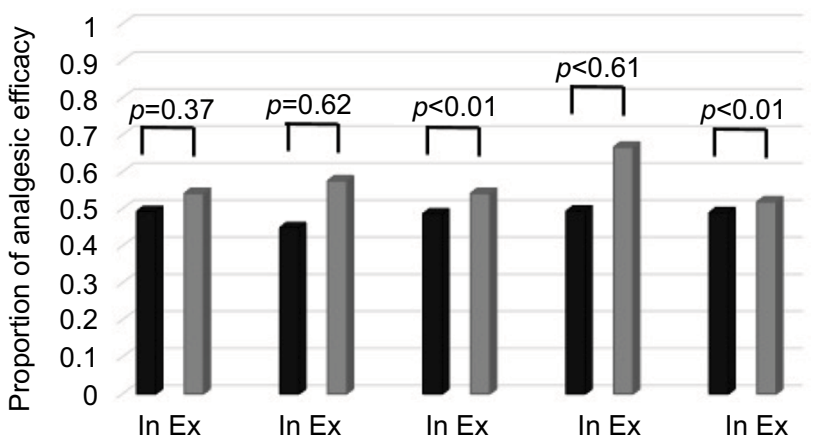

C

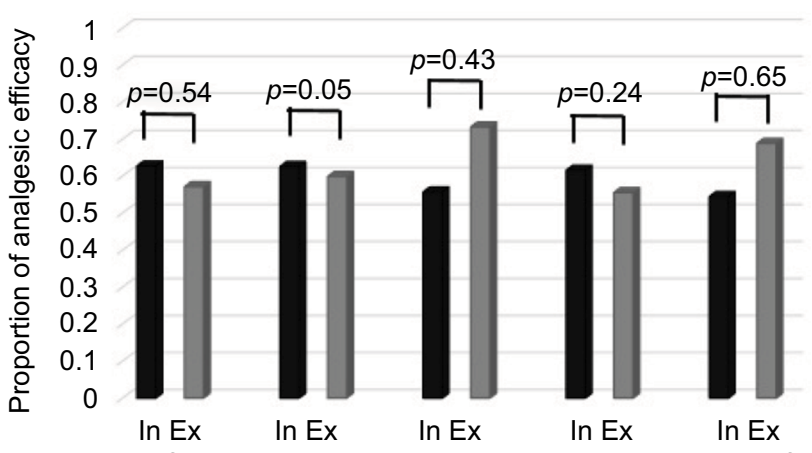

Diclofenac Tramadol Pentazocine Pregabalin Flurbiprofen

Figure 3 Number and efficacy of analgesic drugs for postoperative pain after TS.

Notes: (A) Correlation between the number of analgesic drugs and the NRS score $(r=0.45 \mathrm{I}, p<0.01)$. (B) Comparison of the need for additional diclofenac, tramadol, pentazocine, pregabalin, and flurbiprofen to reduce pain with an NRS score of $\geq 1$. (C) Comparison of the need for additional diclofenac, tramadol, pentazocine, pregabalin, and flurbiprofen to reduce pain with an NRS score of $\geq 3$ upon ambulation dramatically.

Abbreviations: TS, thoracoscopic surgery; NRS, numerical rating scale; In, including the applicable analgesic, Ex, excluding the applicable analgesic.

score reduction of $\geq 3$; tramadol had a borderline tendency of efficacy as an additional prescription ( $p=0.05$; Figure $3 \mathrm{C})$. Multivariate analysis showed that procedures (high surgical stress group; lobectomy/segmentectomy) were significantly influenced in the patients with NRS score of $\geq 3$ upon ambulation (Table 2). 
Table 2 Multivariate analysis in patients with an NRS score of $>3$ on ambulation

\begin{tabular}{llll}
\hline & $\mathbf{p}$ & $\begin{array}{l}\text { Odds } \\
\text { ratio }\end{array}$ & $\mathbf{9 5 \%} \mathbf{C l}$ \\
\hline Age (years) & 0.69 & 0.99 & $0.98-1.01$ \\
Gender (male) & 0.34 & 1.20 & $0.82-1.75$ \\
FEV & 0.73 & 1.00 & $0.98-1.03$ \\
Procedure (lobectomy/segmentectomy) & $<0.0 I^{*}$ & 1.84 & $1.24-2.75$ \\
Bleeding/body weight (>I mL/kg) & 0.48 & 0.81 & $0.44-1.47$ \\
BMI & 0.64 & 0.98 & $0.94-1.04$ \\
Placement of drainage tube & 0.50 & 0.96 & $0.84-1.09$ \\
\hline
\end{tabular}

Note: $* p<0.05$.

Abbreviations: NRS, numerical rating scale; $\mathrm{Cl}$, confidence interval; $\mathrm{FEV}_{1}$, forced expiratory volume in I second; BMI, body mass index.

\section{Discussion}

In this study, we included a study population of $>500$ patients, the largest among previous studies on postoperative pain after TS. Furthermore, we believed that the actual postoperative pain scale was accurately reflected, because only intercostal nerve block was used during the intraoperative course. Our study revealed that pain after TS was not robust in comparison with that after open thoracotomy, at a mean maximum NRS score of 2.73 on ambulation. Although all patients with open thoracotomy used PVB, the mean maximum score on ambulation showed lower score after TS than those after open thoracotomy $(p<0.01)$.

The primary aim of this study was to simplify postoperative pain management, especially in a ward setting in which the protocol for titration of medications for pain control is performed by nursing staff. Certainly, postoperative pain would appear to be less after TS compared with after thoracotomy; nevertheless, surgical trauma during TS may cause postoperative pain that usually lasts for the first 3-4 days until surface reepithelialization occurs. ${ }^{3}$ In our ward, all patients received oral administration, with $180 \mathrm{mg}$ of loxoprofen per day after oral intake of water and either nonsteroidal anti-inflammatory drugs (NSAIDs) or others as additional analgesic drugs were altered by the nursing staff depending on patient requirements. As shown in Figure 3B, additional pentazocine hydrochloride and flurbiprofen significantly reduced pain with an NRS score of $\geq 1$. Therefore, these two drugs might suffice in improving postoperative analgesia; however, additional medications were needed to reduce severe pain (NRS score $\geq 3$ ) on ambulation dramatically.

In this study, patients who needed careful treatment were those who required more than two analgesic prescriptions (26.7\%) and those with moderate-or-severe pain on the day of operation (13.0\%) and upon ambulation (24.4\%). Interestingly, our study postulated that tramadol could be effective as an additional analgesic drug compared with NSAIDs in patients with severe postoperative pain (NRS score $\geq 3$ ), but not as significantly as for general clinical use. This study showed that the surgical stress had a significant influence for this pain (Table 2). According to previous reports on the perioperative period, the use of tramadol was influenced by pain intensity after surgical treatment for breast cancer and after hemicolectomy. ${ }^{10,11}$ Similar to this study, the latter authors did not use a visual analog scale to evaluate the influence of tramadol on postoperative pain intensity. ${ }^{11}$ Therefore, tramadol might be administered for pain prevention or for severe postoperative pain refractory to NSIADs. We believe this to be relevant to the intension given to the local strategy developed by the small population of refractory uncontrolled postoperative pain cases observed.

Tramadol targets two types of postoperative pain: nociceptive and neuropathic pain (intercostal neuralgia). After surgical incision, the first pain sensation is usually nociceptive; therefore, one might question the use of NSAIDs, known to trigger potential intercostal neuralgia. ${ }^{12,13}$ In another prospective and randomized study of 34 patients, pregabalin was considered as an effective and safe drug for the treatment of pain after thoracotomy. ${ }^{14}$ Several authors have described the superior effects of pregabalin for neuropathic pain. ${ }^{14,15}$ In our study, pregabalin was used in few patients, but reduction in pain intensity by NRS was negligible. In addition, we considered the possibility that tramadol exerts similar effects with pregabalin in terms of targeting both nociceptive and neuropathic pain. Despite advances, intercostal neuralgia remains a frequent reason for persistent postsurgical pain 3 months after hospital discharge.${ }^{16}$ Recently, several authors have reported that acute postoperative pain progressed to chronic pain in 11-21 patients after TS. ${ }^{16,17}$ Optimal control of potential intercostal neuralgia might be pivotal to heighten long-term outcome referring to postsurgical satisfaction as a result of preventing persistent pain.

Some limitations of this study should be taken into consideration when interpreting the results. First, the retrospective and single institutional design may have imposed patient selection bias. Second, we used a concise and widely used NRS score to measure postoperative pain. However, it is important to note that NRS score may vary among patients and circumstances. ${ }^{18}$ In addition, the NRS pain score inadequately represents the complexity and idiosyncratic nature of the pain experience or improvements owing to symptom fluctuations. ${ }^{19}$ Nevertheless, our study had several strengths: 1) sufficient statistical power with a larger sample; 2) the frequent assessment of pain scores in order to reflect postoperative pain accurately, especially during the early phase; and 
3) our nursing staff during this study period tended to prefer oral administration or diclofenac suppositories to intravenous drip of pentazocine hydrochloride and flurbiprofen. Further accumulated data must be required to clarify whether our results were representative of those in multiple institutions.

\section{Conclusion}

A small number of patients had moderate-to-severe pain after TS, and these patients were frequently permitted to tolerate postoperative pain without adequate analgesia. Additional pentazocine hydrochloride and flurbiprofen significantly reduced postoperative pain with an NRS score of $\geq 1$, whereas tramadol had a borderline efficacy in reducing postoperative pain with an NRS score of $\geq 3$. Our study suggested the possibility that tramadol contributed to the effective postoperative pain management in the TS patients without PVB or epidural anesthesia.

\section{Acknowledgments}

The abstract of this paper was presented at the ESMO ASIA 2016 by Hitomi Mizuno et al as a poster presentation with interim findings. The poster's abstract was published in "Poster Abstracts" in Annals of Oncology, 2016.

\section{Disclosure}

The authors report no conflicts of interest in this work.

\section{References}

1. Fearon KC, Ljungqvist O, Von Meyenfeldt M, et al. Enhanced recovery after surgery: a consensus review of clinical care for patients undergoing colonic resection. Clin Nutr. 2005;24(3):466-477.

2. Handy JR Jr, Asaph JW, Skokan L, et al. What happens to patients undergoing lung cancer surgery? Outcomes and quality of life before and after surgery. Chest. 2002;122(1):21-30.

3. McKenna RJ Jr, Houck W, Fuller CB. Video-assisted thoracic surgery lobectomy: experience with 1,100 cases. Ann Thorac Surg. 2006;81(2):421-426.

4. Pavlin DJ, Chen C, Penaloza DA, Polissar NL, Buckley FP. Pain as a factor complicating recovery and discharge after ambulatory surgery. Anesth Analg. 2002;95(3):627-634.

5. White PF. The changing role of non-opioid analgesic techniques in the management of postoperative pain. Anesth Analg. 2005;101(5 suppl):5-22.
6. White PF. Multimodal analgesia: its role in preventing postoperative pain. Curr Opin Investig Drugs. 2008;9(1):76-82.

7. Das-Neves-Pereira JC, Bagan P, Coimbra-Israel AP, et al. Fast-track rehabilitation for lung cancer lobectomy: a five-year experience. Eur $J$ Cardiothorac Surg. 2009;36(2):383-391.

8. Goldstraw P, Crowley J, Chansky K, et al; International Association for the Study of Lung Cancer International Staging Committee; Participating Institutions. The IASLC Lung Cancer Staging Project: proposals for the revision of the TNM stage groupings in the for the coming (seventh) edition of the TNM classification of malignant tumors. JThorac Oncol. 2007;2(8):706-714.

9. Kobayashi R, Mori S, Wakai K, et al. Paravertebral block via the surgical field versus epidural block for patients undergoing thoracotomy: a randomized clinical trial. Surg Today. 2013;43(9):963-969.

10. Węgorowski P, Stanisławek A, Domżał-Drzewicka R, et al. The effect of pre-emptive analgesia on the level of postoperative pain in women undergoing surgery for breast neoplasm. Contemp Oncol (Pozn). 2016;20(2):158-164.

11. Wordliczek J, Banach M, Garlicki J, Jakowicka-Wordliczek J, Dobrogowski J. Influence of pre- or intraoperational use of tramadol (preemptive or preventive analgesia) on tramadol requirement in the early postoperative period. Pol J Pharmacol. 2002;54(6):693-697.

12. Rogers ML, Henderson L, Mahajan RP, Duffy JP. Preliminary findings in the neurophysiological assessment of intercostal nerve injury during thoracotomy. Eur J Cardiothorac Surg. 2002;21(2):298-301.

13. Williams EH, Williams CG, Rosson GD, Heitmiller RF, Dellon AL. Neurectomy for treatment of intercostal neuralgia. Ann Thorac Surg. 2008;85(5):1766-1770.

14. Matsutani N, Dejima H, Takahashi Y, Kawamura M. Pregabalin reduces post-surgical pain after thoracotomy: a prospective, randomized, controlled trial. Surg Today. 2015;45(11):1411-1416.

15. Kinney MA, Mantilla CB, Carns PE, et al. Preoperative gabapentin for acute post-thoracotomy analgesia: a randomized, double-blinded, active placebo-controlled study. Pain Pract. 2012;12(3):175-183.

16. Wildgaard K, Ringsted TK, Hansen HJ, Petersen RH, Kehlet H. Persistent postsurgical pain after video-assisted thoracic surgery- an observational study. Acta Anaesthesiol Scand. 2016;60(5):650-658.

17. Handy JR Jr, Asaph JW, Douville EC, Ott GY, Grunkemeier GL, Wu Y. Does video-assisted thoracoscopic lobectomy for lung cancer provide improved functional outcomes compared with open lobectomy? Eur $J$ Cardiothorac Surg. 2010;37(2):451-455.

18. Tandon M, Singh A, Saluja V, Dhankhar M, Pandey CK, Jain P. Validation of a new "objective pain score" Vs. "Numeric Rating Scale" for the evaluation of acute pain: a comparative study. Anesth Pain Med. 2016;6(1):e32101.

19. Hawker GA, Mian S, Kendzerska T, French M. Measures of adult pain: Visual Analog Scale for Pain (VAS Pain), Numeric Rating Scale for Pain (NRS Pain), McGill Pain Questionnaire (MPQ), Short-Form McGill Pain Questionnaire (SF-MPQ), Chronic Pain Grade Scale (CPGS), Short Form-36 Bodily Pain Scale (SF-36 BPS), and Measure of Intermittent and Constant Osteoarthritis Pain (ICOAP). Arthritis Care Res (Hoboken). 2011;63(suppl 11):S240-S252.
Journal of Pain Research

\section{Publish your work in this journal}

The Journal of Pain Research is an international, peer reviewed, open access, online journal that welcomes laboratory and clinical findings in the fields of pain research and the prevention and management of pain. Original research, reviews, symposium reports, hypothesis formation and commentaries are all considered for publication.

\section{Dovepress}

The manuscript management system is completely online and includes a very quick and fair peer-review system, which is all easy to use. Visit http://www.dovepress.com/testimonials.php to read real quotes from published authors. 